\title{
Robust permanence for ecological equations with internal and external feedbacks
}

\author{
Swati Patel $^{1,2,3}$ (D) Sebastian J. Schreiber ${ }^{4}$
}

Received: 25 December 2016 / Revised: 26 August 2017 / Published online: 26 October 2017

(C) The Author(s) 2017. This article is an open access publication

\begin{abstract}
Species experience both internal feedbacks with endogenous factors such as trait evolution and external feedbacks with exogenous factors such as weather. These feedbacks can play an important role in determining whether populations persist or communities of species coexist. To provide a general mathematical framework for studying these effects, we develop a theorem for coexistence for ecological models accounting for internal and external feedbacks. Specifically, we use average Lyapunov functions and Morse decompositions to develop sufficient and necessary conditions for robust permanence, a form of coexistence robust to large perturbations of the population densities and small structural perturbations of the models. We illustrate how our results can be applied to verify permanence in non-autonomous models, structured population models, including those with frequency-dependent feedbacks, and models of eco-evolutionary dynamics. In these applications, we discuss how our results relate to previous results for models with particular types of feedbacks.
\end{abstract}

Keywords Persistence - Robust permanence - Ecological feedbacks - Coexistence · Structured populations $\cdot$ Eco-evolutionary dynamics

Mathematics Subject Classification 92D40 - 92D25 · 37N25

Swati Patel

swpatel@ucdavis.edu

1 Department of Evolution and Ecology and Graduate Group in Applied Mathematics, University of California, Davis, CA 95616, USA

2 Faculty of Mathematics, University of Vienna, Vienna, Austria

3 Department of Mathematics, Tulane University, New Orleans, LA 70115, USA

4 Department of Evolution and Ecology and Center for Population Biology, University of California, Davis, CA 95616, USA 


\section{Introduction}

Understanding when and how species coexist is a fundamental problem in ecology. Permanence theory is a mathematical formalism developed to address this problem for ecological models. Permanence is a particular form of persistence that ensures populations will persist in the face of rare but large perturbations as well as small and frequent perturbations (Schreiber 2006) and hence, is an appropriate notion of coexistence for ecological systems which often experience vigorous shake-ups, rather than gentle stirrings (Jansen and Sigmund 1998). Theory for showing permanence incorporates a variety of standard approaches for characterizing and analyzing dynamical systems including topological approaches, average Lyapunov functions, and measure theoretic approaches. For a review and history on this theory and these approaches see (Hutson and Schmitt 1992; Schreiber 2006; Smith and Thieme 2011). Here, we develop sufficient and necessary conditions for permanence for ecological equations with feedbacks to internal or external variables.

Biologically, many internal and external variables may provide feedbacks on the ecological dynamics of species. By internal variables, we mean factors intrinsic to the populations. For example, this is appropriate for species structured by genotypes of an ecologically-important trait, in which selection affects the frequency of each genotype, or for traits that may change due to phenotypic plasticity. In either case, internal trait changes alter population growth and drive changes in population densities, generating a potential feedback to the trait dynamics. Furthermore, individuals within a population may also be classified into different types (e.g. age or size classes, sex, spatial location) and this may influence their growth as well as the growth of the populations they interact with. In particular, this population structure is important for species with life stages, between which individuals can transition, or species living in patchy landscapes, between which individuals can disperse. By external variables, we mean dynamic variables extrinsic to the populations that influence survivorship, growth rates and reproductive rates. For example, environmental variables such as precipitation or temperature which vary in time or the constructed habitats of ecosystem engineering species often influence these demographic rates. These internal and external variables may influence coexistence and motivate us to characterize permanence in models that account for general feedbacks with these variables.

Permanence has been studied for general dynamical systems, abstracting beyond classical ecological models (Hutson 1984b; Butler and Waltman 1986; Hutson 1988; Garay 1989; Hale and Waltman 1989). For example, Garay (1989) characterized permanence using Morse decompositions and Hutson (1984b, 1988), by extending work of Hofbauer (1981), found a characterization using so-called average Lyapunov functions. Combining these approaches, Garay and Hofbauer (2003) provided sufficient conditions for robust permanence for ecological equations in the standard form

$$
\frac{d x_{i}}{d t}=x_{i} f_{i}(x) \quad i=1 \ldots n
$$

where $x_{i}$ is population densities and $f_{i}$ is the per-capita growth rate of population $i$. Robust permanence ensures that permanence holds following small perturbations of 
the per-capita growth rate equations (Schreiber 2006). Garay and Hofbauer (2003) and Schreiber (2000) showed that robust permanence can be characterized in terms of the average per-capita growth rates of missing species for trajectories of (1) on the extinction set. These ecological equations, however, assume that the per-capita growth rates only depend on the densities of the species, ignoring internal differences amongst individuals in the populations and external influences.

That internal and external variation exists is indisputable; no two individuals in a population are identical and environmental conditions always vary in time. From a modeling perspective, the ubiquity of both varying internal and external variables requires careful choice on when and how to include these variables. In some familiar cases, feedbacks are implicitly modeled, such as in some models of interspecific competition with species competing for a limited resource (Schoener 1976) or predator-prey models with prey switching behavior (Hutson 1984a; van Baalen et al. 2001). In other cases, feedback variables are explicitly modeled and this allows them to have their own dynamics. Hence, an important scientific goal is to determine when and how these feedbacks impact populations and communities. Some studies have examined permanence in models with specific types of internal or external feedbacks, such as for internally structured populations (Hofbauer and Schreiber 2010) or environmental variation (Gatica and So 1988; Schreiber et al. 2011a; Roth et al. 2017). However, there is no general framework for dealing explicitly with both internal and external variables.

In the present paper, we derive sufficient conditions for robust permanence in a general model of interacting populations with internal and external feedbacks and demonstrate how it generalizes and extends prior results of models accounting for these feedbacks. Our main permanence results build on average Lyapunov functions developed by Garay and Hofbauer (2003). In Sect. 2, we introduce the general model and describe our main assumptions. Then, we state our sufficient and necessary criteria for permanence and robust permanence in Sects. 3 and 4, respectively. In Sect. 5, we apply our main theorem to three distinct models with feedbacks from the environment, population structure, and trait evolution to demonstrate its broad applicability and the importance of internal and external feedbacks on species coexistence.

\section{Model and terminology}

We extend model (1) to incorporate internal and external feedbacks. We suppose that $n$ populations are interacting in a community and that population $i$ has density $x_{i}$, with $i=1 \ldots n$. Interactions can include competition, predation as well as mutualisms. For each population $i$, the per-capita growth rate, $f_{i}$, depends on the densities of all the species it interacts with, as well as on another set of $m$ variables. These $m$ variables can represent a combination of internal factors, such as the stages in a life cycle of a population, and external factors, such as temperature or another environmental variable. Each of these factors is represented quantitatively by $y=\left(y_{1}, \ldots, y_{m}\right) \in$ $K \subset \mathbb{R}^{m}$, and can also change due to feedbacks with the population densities as well as all $m$ factors. Altogether, the dynamics in this fairly general ecological scenario can be expressed with the differential equation model 


$$
\begin{aligned}
\frac{d x_{i}}{d t} & =x_{i} f_{i}(x, y) & i & =1 \ldots n \\
\frac{d y_{j}}{d t} & =g_{j}(x, y) & j & =1 \ldots m
\end{aligned}
$$

where $x=\left(x_{1}, \ldots, x_{n}\right) \in \mathbb{R}_{+}^{n}=[0, \infty)^{n}$ is the vector of population densities. Note that both $f$ and $g$ can depend on both $x$ and $y$, capturing the potential feedback between the population densities and the other dynamic variables. The model form is quite general and can apply to a variety of types of feedbacks as illustrated in Sect. 5, where we apply our theorem to different biological scenarios.

Let $S=\mathbb{R}_{+}^{n} \times K$ be the state space for (2). We let $z . t$ denote the solution to (2) for initial condition $z=(x, y) \in S$. For any set $Z \subseteq S$ and $I \subseteq \mathbb{R}_{+}$, let $Z . I=\{z . t \mid t \in I, z \in Z\}$.

We make the following standing assumptions:

S1: $x_{i} f_{i}$ and $g_{j}$ are locally Lipschitz functions, and

S2: there exists a compact set $Q \subseteq S$ such that $Q .[0, \infty) \subseteq Q$ and z.t $\in Q$ for $t$ sufficiently large for all $z \in S$.

As we demonstrate in Sect. 5, both assumptions hold for many biological models. The first assumption ensures that solutions to (2) locally exist and are unique. The second assumption corresponds to the biological reality that population densities do not grow without bound.

The extinction set $S_{0}:=\left\{z=(x, y) \in S \mid \prod_{i=1}^{n} x_{i}=0\right\}$ is the set which has at least one species extinct, i.e., with density equal to zero. Observe from model (2) that for any initial condition in $z \in S_{0}$, z.t stays in $S_{0}$ for all time, capturing the "no cats, no kittens" principle of closed ecological systems.

To use our model to identify the conditions that ensure community coexistence, we must formulate a precise notion of coexistence. The importance of understanding coexistence in ecology has inspired many different notions of coexistence (Schreiber 2006). Here, we use the notion of permanence, which ensures that there is a positive population density that each species eventually stays above provided all populations are initially present. Precisely, model (2) is permanent if there is a $\beta>0$ such that for all $z \in S \backslash S_{0}$

$$
\liminf _{t \rightarrow \infty} x_{i}(t) \geq \beta \quad \text { for } i=1,2, \ldots, n
$$

for all $i$, where $x_{i}(t)$ is the $i^{t h}$ component of $z . t=(x, y) . t$.

Permanence implies that if all the species are initially coexisting, then they will continue to coexist, despite rare but large perturbations or frequent small perturbations (Schreiber 2006). In the next section, we present the main theorems, which establishes sufficient and necessary conditions for permanence for models of the form (2).

Before stating our main theorem, we introduce some terminology. The $\omega$-limit set of a set $Z \subset S$ is $\omega(Z):=\cap_{t \geq 0} \overline{Z .[t, \infty)}$ and the $\alpha$-limit set is $\alpha(Z):=\cap_{t \leq 0} \overline{Z .(-\infty, t]}$. A set $Z \subset S$ is invariant if $Z . \mathbb{R}=Z$. A compact invariant set $Z$ is isolated if there exists a closed neighborhood $U$ such that for all $z \in U \backslash Z$ there is a $t$ such that $z$.t $\notin U$. For any compact invariant set $Z$, a subset $A \subset Z$ is called an attractor in $Z$ if there is a neighborhood $U$ of $A$ such that $\omega(U \cap Z)=A$. The dual repeller to an attractor 
$A$ in $Z$ is $R(A)=\{z \in Z \mid \omega(z) \cap A=\emptyset\}$ and $A, R(A)$ are called attractor-repeller pairs. The global attractor is $\omega(Q)$.

\section{Permanence theorem}

We take advantage of a characterization of permanence involving Morse decompositions. Roughly, a Morse decomposition for a compact invariant set is a finite number of disjoint invariant subsets, called Morse sets, ordered in such a way that the flow tends to move from sets of higher order to lower order. More precisely,

Definition 1 A collection of sets $\mathcal{M}=\left\{M_{1}, M_{2}, \ldots, M_{\ell}\right\}$ is a Morse decomposition for a compact invariant set $\Gamma$ if $M_{1}, M_{2}, \ldots, M_{\ell}$ are pairwise disjoint, isolated invariant compact sets, called Morse sets, such that for every $z \in \Gamma \backslash \cup_{k=1}^{\ell} M_{k}$ there are integers $i<j$ such that $\omega(z) \subset M_{i}$ and $\alpha(z) \subset M_{j}$.

For a compact invariant set $\Gamma$, Morse decompositions always exist but are not necessarily unique. Trivially, one Morse decomposition for $\Gamma$ is $\{\Gamma\}$. However, more refined Morse decompositions are typically more useful. In our main theorem, we use Morse decompositions to decompose the global attractor on the extinction set and define conditions on the Morse sets that give permanence for (2).

Theorem 1 Let $\mathcal{M}=\left\{M_{1}, M_{2}, \ldots M_{\ell}\right\}$ be a Morse Decomposition for $S_{0} \cap \Gamma$ where $\Gamma$ is the global attractor for (2). If, for each $M_{k} \in \mathcal{M}$, there exists $p_{k 1}, \ldots, p_{k n}>0$ such that for every $z \in M_{k}$, there is a $T_{z}$ such that

$$
\sum_{i=1}^{n} p_{k i} \int_{0}^{T_{z}} f_{i}(z . t) d t>0
$$

then (2) is permanent.

In words, if, for each Morse set, the weighted combination (weights are $p_{k i}$ ) of the per-capita growth rates over some time period is positive from every point in the Morse set, then there is permanence. Moreover, there is an $A \subset \Gamma$ such that $S_{0} \cap \Gamma$ is $R(A)$.

Given the Morse decomposition for $S_{0} \cap \Gamma$, we can show the following partial converse

Corollary 1 For each Morse set $M_{k}$, if there is a $p_{k 1}, \ldots, p_{k n}>0$ such that for every $z \in M_{k}$, there is a $T_{z}>0$ such that

$$
\sum_{i} p_{k i} \int_{0}^{T_{z}} f_{i}(z . t) d t<0
$$

then (2) is not permanent and, more strongly, $S_{0} \cap \Gamma$ is an attractor in $\Gamma$. 
The proof of this partial converse follows from applying Theorem 1 to the reverse time flow in $\Gamma$ to show that $S_{0} \cap \Gamma$ is a repeller in reverse time.

Intuitively, for permanence to hold, population densities near extinction have to increase. Lyapunov functions are functions that increase along solutions on some subset of the state space, and are used to characterize local and global stability of invariant sets, including equilibria. Extending beyond equilibria, "average Lyapunov functions", introduced by Hofbauer (1981), are functions that increase on average along solutions. These can apply to more complex invariant sets, which are common in many population models of the form (1).

In Appendix 1, we prove Theorem 1. In particular, we define "good" average Lyapunov functions (GALFs), as introduced by Garay and Hofbauer (2003), and then prove that the existence of a GALF on each Morse set gives permanence. Then, we show that the condition in Theorem 1 on the weighted per-capita growth functions implies the existence of a GALF in the standard form

$$
P(x, y)=\prod_{i=1}^{n} x_{i}^{p_{i}}
$$

for some vector $p$ with $p_{i}>0$. The standard form (4) is zero everywhere on the extinction set, positive everywhere not on the extinction set and has time derivative

$$
\dot{P}=P \sum_{i=1}^{n} p_{i} f_{i}
$$

This time derivative gives a convenient relationship between the function and the per-capita growth rates, $f_{i}$, of each of the species. Feedbacks with the internal or external variable $y$ will affect the per-capita growth rates, thereby influencing the existence and construction of Lyapunov functions.

\section{Robust permanence}

Population models are always approximations of reality. In the words of Conley (1978), "if such rough equations are to be of use, it is necessary to study them in rough terms". In line with this, Hutson and Schmitt (1992) introduced robust permanence, i.e. that permanence holds even with sufficiently small perturbations to the growth functions $f_{i}$ and Schreiber (2000) subsequently provided conditions for robust permanence for (1) using a measure theoretic approach. More recently, Garay and Hofbauer (2003) showed robust permanence for (1) using GALFs. We use this method to extend our permanence result to robust permanence, with respect to perturbations in both the growth functions and the feedback dynamics. 
Suppose we have a perturbed system

$$
\begin{aligned}
\frac{d x_{i}}{d t} & =x_{i} \widetilde{f}_{i}(x, y) \\
\frac{d y_{j}}{d t} & =\tilde{g}_{j}(x, y)
\end{aligned}
$$

Let $\widetilde{z . t}$ denote the solution of (5) with initial condition $z \in S$ and analogously, for set $Z \subseteq S$ and $I \subseteq \mathbb{R}_{+}, \widetilde{Z . I}=\{\widetilde{z . t} \mid t \in I, z \in Z\}$. Also, let $\widetilde{\omega}(Z), \widetilde{\alpha}(Z)$ denote the $\omega, \alpha$-limit set for $(5)$, respectively. Let $Q$ be as defined previously. We define $(\widetilde{f}, \widetilde{g})$ to be a $(\delta, Q)$-perturbation of (2) if

R1: $\left|\widetilde{f}_{i}(x, y)-f_{i}(x, y)\right|<\delta$ and $\left|\tilde{g}_{j}(x, y)-g_{j}(x, y)\right|<\delta$ for all $i, j$ and for all $(x, y) \in Q$

R2: $x_{i} \widetilde{f}_{i}$ and $\widetilde{g_{j}}$ are all locally Lipschitz continuous, and

R3: $\widetilde{Q . \mathbb{R}_{+}} \subseteq Q$ and for all $z \in S, \widetilde{z . t} \in Q$ for $t$ sufficiently large.

Denote the set of all $(\delta, Q)$-perturbations as $\Delta(\delta, Q)$. This set contains differential equation models that are close to the unperturbed model (2), which have solutions that eventually enter the compact set $Q$.

Definition 2 (2) is robustly permanent if there is a $\delta>0$ and $\beta>0$ such that for all $(\tilde{f}, \widetilde{g}) \in \Delta(\delta, Q),(3)$ holds for all $z \in S \backslash S_{0}$.

Theorem 2 The conditions in Theorem 1 imply robust permanence.

To show this, we apply a result from Hirsch et al. (2001) to show permanence of $(\tilde{f}, \tilde{g}) \in \Delta(\delta, Q)$ with a uniform lower bound $\beta$. A proof is given in Appendix 2. It is worth noting that permanence does not in general imply robust permanence; $\dot{x}=x^{2}(1-x)$ is permanent but not robustly permanent. Hofbauer and Schreiber (2004) show that robust permanence is not generic among permanent ecological equations.

\section{Applications}

The main results developed here are applicable to a broad range of internal and external feedbacks. In this section, we discuss permanence in models with external environmental, internal structural and evolutionary feedbacks, which illustrate the utility of the main theorem. In the first example, we apply our result to show how external environmental fluctuations can enable coexistence amongst competing species in the form of robust permanence. In the second example, we demonstrate how existing permanence conditions from Hofbauer and Schreiber (2010) for models with internal population structure, i.e., the partitioning of a whole population into distinct types, can be reproduced using our framework. Then we give an example of a sexually-structured population model to which the existing result from Hofbauer and Schreiber (2010) does not apply, emphasizing the utility of our result to structured models. Finally, in the third example, we apply the result to an example of an ecological model with the evolution of a quantitative trait as the internal feedback, demonstrating how our 
results apply to models of eco-evolutionary dynamics. Altogether, these applications highlight how Theorem 1 unifies some existing permanence results and how it enables us to determine when there is permanence in population models with a variety of feedbacks.

\subsection{Environmental fluctuations}

Population dynamics are often influenced by time-varying environmental factors, such as seasonal fluctuations in temperature and rain fall or other weather patterns. When environmental factors influence populations' growth rate, this may affect persistence of the community. Non-autonomous differential equations, with time-varying parameters, are commonly used to account for the temporal changes in growth rates (e.g. Vance and Coddington 1989; Zhao 2001; Smith and Thieme 2011). These give the differential equation

$$
\frac{d x_{i}}{d t}=x_{i} f_{i}(x, t) \quad i=1 \ldots n
$$

where the per-capita growth rates depend on time.

Non-autonomous models can be formulated into our model form (2) when the environmental factors can be modeled as a solution of an autonomous differential equation $\frac{d y}{d t}=g(y)$. Then (6) becomes

$$
\begin{array}{ll}
\frac{d x_{i}}{d t}=x_{i} f_{i}(x, y) & i=1 \ldots n \\
\frac{d y_{j}}{d t}=g_{j}(y) & j=1 \ldots m
\end{array}
$$

To apply our main theorem, $y$ must remain in a compact set $K \subset \mathbb{R}^{m}$. Biologically, there is no mutual feedback between $y$ and $x$, which is appropriate when $y$ represents environmental factors, such as weather, that are independent of the population densities. Model (7) is a special case of a skew product flow, which are commonly used for studying non-autonomous flows (Zhao 2001; Mierczyński et al. 2004).

To illustrate how our results can be applied to non-autonomous systems, we first prove a general, algebraically verifiable condition for non-autonomous Lotka-Volterra systems where only the "intrinsic" per-capita growth rates fluctuate. Indeed, for these Lotka-Volterra systems permanence conditions are equivalent to an autonomous Lotka-Volterra system with the fluctuating intrinsic rate of growth replaced by an averaged intrinsic rate of growth. When the interaction coefficients fluctuate, however, this simplification is no longer possible. We illustrate verifying our permanence condition in this latter case for a Lotka-Volterra system with two competing species.

For the general result, consider a non-autonomous Lotka-Volterra system of the form

$$
\begin{aligned}
& \frac{d x}{d t}=x \circ(A x+b(y)) \\
& \frac{d y}{d t}=g(y)
\end{aligned}
$$


where $\circ$ denotes component-wise multiplication i.e., the Hadamard product. The matrix $A=\left(a_{i j}\right)$ corresponds to the matrix of per-capita species interaction strengths and the vector $b(y)$ corresponds to the intrinsic per-capita growth rates as a function of the "environmental" state $y$. As $y$ doesn't depend on $x$, we write $y . t$ as the solution of $\frac{d y}{d t}=g(y)$ with initial condition $y \in K$.

For simplicity, we assume the dynamics of $y$ on $K$ are uniquely ergodic, i.e., there exists a Borel probability measure $\mu$ on $K$ such that

$$
\bar{h}:=\lim _{t \rightarrow \infty} \frac{1}{t} \int_{0}^{t} h(y . s) d s=\int h(y) \mu(d y)
$$

for all $\mu$-integrable functions $h: K \rightarrow \mathbb{R}$ satisfying $\int|h(x)| \mu(d x)<\infty$. In particular, let $\bar{b}=\left(\overline{b_{1}}, \ldots, \overline{b_{n}}\right)$ be the temporal averages of the intrinsic rates of growth. Using these averages, we prove the following two results.

Proposition 1 Assume that (8) satisfies assumption $\mathbf{S} 2$ and that the dynamics of $y$ on $K$ are uniquely ergodic. If there exist $p_{1}, \ldots, p_{n}>0$ such that

$$
\sum_{i} p_{i}\left(\sum_{j} a_{i j} x_{j}+\overline{b_{i}}\right)>0
$$

for any $x \in \mathbb{R}_{+}^{n}$ satisfying $\prod_{i} x_{i}=0$ and $\sum_{j} a_{i j} x_{j}=-\overline{b_{i}}$ whenever $x_{i}>0$, then (8) is robustly permanent.

The proof of this proposition is in Appendix 3.

Proposition 2 If there is no $x \in \mathbb{R}_{+}^{n}$ such that $\sum_{j} a_{i j} x_{j}=-\overline{b_{i}}$ with $x_{i}>0$ for all $i$, then $\omega(z) \subset S_{0}$ for all $z \in S \backslash S_{0}$.

Proof Following the proof of Theorem 5.2.1 in Hofbauer and Sigmund (1998), there exists a $p$ such that $\sum_{i} p_{i}\left(\sum_{j} a_{i j} x_{j}+\overline{b_{i}}\right)>0$ for all $x \in \mathbb{R}_{+}^{n}$. Let $V(z)=$ $\sum_{i} p_{i} \log \left(x_{i}\right)$ for all $z=(x, y) \in S \backslash S_{0}$. Then $\frac{d V}{d t}=\sum_{i} p_{i}\left(\sum_{j} a_{i j} x_{j}(t)+b_{i}(y . t)\right)$. Now, suppose there is a $z \in S \backslash S_{0}$ with $\omega(z) \subset S \backslash S_{0}$. Then, by compactness, there is a $z^{*} \in \omega(z)$ such that $V$ is maximized on $\omega(z)$. Also, since $z^{*} \in S \backslash S_{0}$, there is a $T>0$ such that $\frac{1}{T} \int_{0}^{T} \frac{d}{d t} V\left(z^{*} . s\right) d t>0$ but this contradicts the existence of a maximum. It follows that for all $z \in S \backslash S_{0}, \omega(z) \not \subset S \backslash S_{0}$ and $\omega(z) \cap S_{0} \neq \emptyset$. Then, by the Zubov-Ura-Kimura theorem (Garay and Hofbauer 2003), $\omega(z) \subset S_{0}$.

Hence, when environmental variation drives fluctuations in intrinsic growth rates, their effects can be averaged in time to determine permanence. On the contrary, we will show that if interaction coefficients fluctuate, then permanence may hold, even if predictions from averaging these coefficients in time suggest otherwise.

To demonstrate this explicitly, we consider a modified version of the autonomous model from Volterra (1928) of two species competing for a single limiting resource. Let $x_{1}$ and $x_{2}$ be the densities of two species competing for a limited resource, $R$. Suppose the death rate and resource use of species $i$ depend on a changing environmental state $y$ 
so that the intrinsic death rate $d_{i}(y)$ and the interaction coefficients $a_{i}(y)$ are functions of $y$. The model from Volterra (1928) becomes

$$
\begin{aligned}
\frac{d x_{1}}{d t} & =x_{1}\left(c a_{1}(y) R-d_{1}(y)\right) \\
\frac{d x_{2}}{d t} & =x_{2}\left(c a_{2}(y) R-d_{2}(y)\right) \\
\frac{d y}{d t} & =g(y) \\
R & =\max \left\{J-a_{1}(y) x_{1}-a_{2}(y) x_{2}, 0\right\}
\end{aligned}
$$

where $c$ is the efficiency with which both species convert the resource into new individuals and $J$ is the maximum amount of resource available and this is instantly reduced by the competitors. We assume that the dynamics of $y$ are uniquely ergodic on a compact set $K$. This model is appropriate for species in which resource use or death rate change with the seasons or a fluctuating environment.

In the constant environment model $(g(y)=0)$, Volterra (1928) showed that if $\frac{d_{1}(y)}{a_{1}(y)}<\frac{d_{2}(y)}{a_{2}(y)}<J c$, species 1 will exclude species 2 : $\lim _{t \rightarrow \infty} x_{2}(t)=0$ for any initial condition $z=\left(x_{1}, x_{2}, y\right)$ satisfying $x_{1} x_{2}>0$. This is commonly referred to in the ecological literature as the $R^{*}$ rule (Tilman 1980) and is a mathematical formulation of the competitive exclusion principle, which asserts that two competing species for the same resource cannot coexist, if other ecological factors are constant (Gause 1934; Hardin 1960).

Environmental fluctuations that lead to time-varying parameters might affect the coexistence of two species competing for the same resource. Proposition 2 implies that if only the per-capita death rates vary, then the competitive exclusion principle still holds. However, when the resource use rates vary coexistence is possible. Specifically, suppose that species $i$ uses the resource at a maximal rate for some compact subset of environmental states $K_{i} \subset K$ so that $a_{i}(y)=1$ and $a_{j}(y)=0$ for $y \in K_{i}, i \neq$ $j$. To allow for temporal partitioning of resource use, we assume that these sets of environmental states are disjoint i.e. $K_{1} \cap K_{2}=\emptyset$. Let $k_{i}=\lim _{t \rightarrow \infty} \frac{1}{t} \int_{0}^{t} \mathbb{1}_{K_{i}}(y . s) d s$ be the average time spent in environmental state $K_{i}$, where $\mathbb{1}_{K_{i}}: K \rightarrow \mathbb{R}$ is the indicator function with $\mathbb{1}_{K_{i}}(y)=1$ for $y \in K_{i}$ and 0 otherwise. Furthermore, assume that $d_{i}(y)>\epsilon$ for some $\epsilon>0$ and for all $y$ and $i=1,2$. For example, this might model the dynamics of winter annual plants in the Sonoran desert that use water following winter rains, while summer annuals tend to do so during summer (Smith et al. 1997).

Theorem 3 If $c J k_{i}>\overline{d_{i}}$ for $i=1,2$, then (10) is robustly permanent.

Proof First, note that (10) satisfies S2 with $Q=\left\{\left[0, \frac{c J^{2}}{\epsilon}\right] \times K\right\}$, as $\frac{d x_{i}}{d t}<0$ whenever $x_{i}>\frac{c J^{2}}{\epsilon}$.

Next, we show that each species persists on its own when the other species is absent. Consider the single species $i$ model 


$$
\begin{aligned}
\frac{d x_{i}}{d t} & =x_{i}\left(c a_{i}(y)\left(\max \left\{J-a_{i}(y) x_{i}, 0\right\}\right)-d_{i}(y)\right) \\
\frac{d y}{d t} & =g(y)
\end{aligned}
$$

on $S^{i}=\mathbb{R}_{+} \times K$ with extinction set $S_{0}^{i}=\{0\} \times K . \mathcal{M}=\left\{S_{0}^{i}\right\}$ is a Morse decomposition for $\Gamma_{i} \cap S_{0}^{i}$, where $\Gamma_{i}$ is the global attractor for (11). Then, for all $z \in S_{0}^{i}$,

$$
\lim _{t \rightarrow \infty} \frac{1}{t} \int_{0}^{t} f_{i}(0, y . s) d s=\lim _{t \rightarrow \infty} \frac{1}{t} \int_{0}^{t}\left(c a_{i}(y . s) J-d_{i}(y . s)\right) d s>c J k_{i}-\bar{d}_{i}>0
$$

By Theorem 1, (11) is permanent. Let $A_{i} \subset S^{i} \backslash S_{0}^{i}$ be the attractor in $\Gamma_{i}$.

Now, consider (10). Let $M_{3}=\{0\} \times\{0\} \times\{K\}$ and $M_{i}=A_{i}$ for $i=1,2$. Then, $\mathcal{M}=\left\{M_{3}, M_{2}, M_{1}\right\}$ is a Morse decomposition for $S_{0} \cap \Gamma$, where $\Gamma$ is a global attractor for $(10)$. With $p=(1,1)$, the inequality in Theorem 1 is satisfied for Morse set $M_{3}$. For $i=1,2$,

$$
\lim _{t \rightarrow \infty} \frac{1}{t} \int_{0}^{t} f_{i}(z . s) d s=0
$$

and

$$
\lim _{t \rightarrow \infty} \frac{1}{t} \int_{0}^{t} f_{j}(z . s) d s>c J k_{1}-\overline{d_{1}}>0
$$

for $j \neq i$, for all $z \in M_{i}$. Then $p$ satisfies the inequality in Theorem 1 for $M_{i}$. Finally, by Theorem 2, (10) is robustly permanent.

This result implies that even if species 1 is on average a stronger resource competitor, i.e., $\frac{\bar{d}_{1}}{c \bar{a}_{1}}<\frac{\bar{d}_{2}}{c \bar{a}_{2}}$, it may not always exclude species 2 . Temporal differences in resource use enable weaker competitors to coexist with stronger competitors. The condition in Theorem 3 suggests that when per-capita death rates are high, the species needs a longer time period to maximally acquire the resource to ensure permanence. Furthermore, the more resource that is available (greater $J$ ), the shorter this time period can be, all else being equal. This is an example of the storage effect mechanism of coexistence: species have different environmental time periods that are good for growth and are able to survive through time periods bad for growth (Chesson and Warner 1981; Chesson 1994).

\subsection{Structured populations}

Individual variation that gives rise to intraspecific differences in demographic rates and species interactions can alter community dynamics and hence, persistence (Moll and Brown 2008; Bolnick et al. 2011; Fujiwara et al. 2011; van Leeuwen et al. 2014). One form of structured population models account for this individual variation by partitioning populations into discrete types, e.g. size classes, spatial location, and 
gender. For example, Hofbauer and Schreiber (2010) considered models of interacting, structured populations of the form

$$
\frac{d u_{i}}{d t}=A^{i}(u) u_{i}
$$

where $u_{i}=\left(u_{i 1}, \ldots, u_{i m_{i}}\right)$ is a vector of densities for the $m_{i} \geq 1$ subpopulations of species $i, u=\left(u_{1}, u_{2}, \ldots, u_{n}\right)$ is the state of the entire community, and $A^{i}(u)=$ $\left(a_{j k}^{i}(u)\right)_{j, k}$ are $n_{i} \times n_{i}$ matrices with non-negative off-diagonal entries and the sign structure of an irreducible matrix that only depends on $i$. First, we show how our result reproduces a previous result from Hofbauer and Schreiber (2010) for permanence in structured population models. Second, through a sexually-structured model, we illustrate how our result applies to models that prior results do not.

\subsubsection{Reproduce results from Hofbauer and Schreiber (2010)}

Assume that the semi-flow defined by equation (12), with solutions $u$.t for initial condition $u$, has a global attractor $\Gamma$. To characterize robust permanence of these equations, Hofbauer and Schreiber (2010) used dominant Lyapunov exponents that characterize the long-term growth rates of each of the species. To define the exponents for species $i$, consider the linear skew product flow on $\Gamma \times \mathbb{R}^{m_{i}}$ defined by $(u \cdot t, v . t)=$ $\left(u . t, B_{i}(t, u) v\right)$ where $Y(t)=B_{i}(t, u)$ is the solution to $Y^{\prime}(t)=A_{i}(u . t) Y(t)$ with $Y(0)$ equal to the identity matrix. The assumption that $A_{i}$ is irreducible with nonnegative off diagonal entries implies that $B_{i}(t, u) \mathbb{R}_{+}^{m_{i}} \subset(0, \infty)^{m_{i}}$ for all $u$ and $t>0$ (e.g., Smith 1995). Ruelle (1979, Prop.3.2) provides a non-autonomous form of the Perron-Frobenius Theorem: there exist continuous maps $v_{i}, w_{i}: \Gamma \rightarrow \mathbb{R}_{+}^{m_{i}}$ with $\left\|v_{i}(u)\right\|=\left\|w_{i}(u)\right\|=1$, where $\|v\|=\sum_{i}\left|v_{i}\right|$, such that

- The line bundle $E_{i}(u)$ spanned by $v_{i}(u)$ is invariant, i.e., $E_{i}(u . t)=B_{i}(u, t) E_{i}(u)$ for all $t \geq 0$.

- The vector bundle $F_{i}(u)$ perpendicular to $w_{i}(u)$ is invariant i.e., $F_{i}(u . t)=$ $B_{i}(u, t) F_{i}(u)$ for all $t \geq 0$.

- There exist constants $\alpha>0$ and $\beta>0$ such that

$$
\left\|B_{i}(t, u)\left|F_{i}(u)\|\leq \alpha \exp (-\beta t)\| B_{i}(t, u)\right| E_{i}(u)\right\|
$$

for all $u \in \Gamma$ and $t \geq 0$.

In light of (13), $v_{i}(u)$ can be viewed as the community state-dependent "stable stage distribution" of species $i$ for the linearized dynamics given by $Y^{\prime}(t)=A_{i}(u . t) Y(t)$. Specifically, (13) implies that for any $\tilde{v} \in(0, \infty)^{m_{i}}, Y(t) \tilde{v} /\|Y(t) \tilde{v}\|-v(u . t)$ converges to zero as $t \rightarrow \infty$. Similarly, $w_{i}(u)$ can be interpreted as the community state-dependent vector of "reproductive values" for the stages of species $i$. Stages with larger entries in $w_{i}(u)$ contribute more to the long-term growth rate of species $i$. 
Hofbauer and Schreiber (2010) defined the average per-capita growth rate of species $i$ given the initial community state $u$ as

$$
r_{i}(u)=\limsup _{t \rightarrow \infty} \frac{1}{t} \int_{0}^{t} w_{i}(u . s)^{T} A_{i}(u . s) v_{i}(u . s) d s
$$

where $w^{T}$ denotes the transpose of a vector $w$. We derive the following theorem of Hofbauer and Schreiber (2010) as a corollary of Theorem 1.

Theorem 4 Let $\left\{M_{1}, \ldots, M_{\ell}\right\}$ be a Morse decomposition for $S_{0} \cap \Gamma$. If for each $M_{k}$ there exists $p_{k 1}, \ldots, p_{k n}>0$ such that

$$
\sum_{i} p_{k i} r_{i}(u)>0
$$

for all $u \in M_{k}$, then system (12) is robustly permanent.

Proof To prove Theorem 4 using our framework, we introduce the following change of variables:

$$
x_{i}=\sum_{j} u_{i j} \text { and } y_{i j}=u_{i j} / x_{i}
$$

In this coordinate system, equation (12) becomes

$$
\begin{aligned}
& \frac{d x_{i}}{d t}=x_{i} \sum_{j, k} b_{j k}^{i}(x, y) y_{i k}=: x_{i} f_{i}(x, y) \text { where } b_{j k}^{i}(x, y)=a_{j k}^{i}(u) \\
& \frac{d y_{i j}}{d t}=\left(\sum_{k} b_{j k}^{i}(x, y) y_{i k}-y_{i j} f_{i}(x, y)\right)=: g_{i j}(x, y) .
\end{aligned}
$$

The state space for equation (15) is $\tilde{S}=\mathbb{R}_{+}^{n} \times \Delta_{m_{1}} \times \ldots \Delta_{m_{n}}$ where $\Delta_{k}=\left\{y \in \mathbb{R}_{+}^{k}\right.$ : $\left.\sum_{j} y_{j}=1\right\}$ is the $k-1$ dimensional simplex. Let $\tilde{\Gamma} \subset \tilde{S}$ and $\left\{\tilde{M}_{k}\right\}_{k=1}^{\ell}$ be the global attractor $\Gamma$ and the Morse decomposition $\left\{M_{k}\right\}_{k=1}^{\ell}$, respectively, for equation (12) in this coordinate system.

Fix an element $\tilde{M}_{k}$ of the Morse decomposition and $z=(x, y) \in \tilde{M}_{k}$. Let $u$ be $z$ in the original coordinate system. Proposition 1 of Hofbauer and Schreiber (2010) implies that

$$
r_{i}(u)=\liminf _{t \rightarrow \infty} \frac{1}{t} \int_{0}^{t} f_{i}(z . s) d s .
$$

By the assumption of the theorem statement,

$$
\sum_{i} p_{i} r_{i}(u)>0
$$


Hence, we can choose $T_{z}>0$ such that

$$
\sum_{i} p_{i} \int_{0}^{T_{z}} f_{i}(z . s) d s>0 .
$$

Applying Theorem 1 completes the proof.

The change of variables from (12) to (15) demonstrates how structured populations can be reformulated into our general framework and reproduce results from Hofbauer and Schreiber (2010).

\subsubsection{Sexually structured populations}

Our main permanence result applies to structured models that Hofbauer and Schreiber (2010) does not. In particular, permanence results from Hofbauer and Schreiber (2010) do not apply to models in which growth depends on the frequency of types in the populations.

As an example, we consider a rock-paper-scissors three-species competition model, in which each species is sexually-structured such that reproduction depends on the frequencies of males and females. Let $m_{i}$ be the density of males and $f_{i}$ the density of females for species $i$. Following Caswell and Weeks (1986), we assume that there is a harmonic mating function in which case the rate at which females and males are produced (assuming a 50-50 primary sex-ratio) is

$$
b \frac{m_{i} f_{i}}{f_{i}+m_{i}}
$$

where $2 b$ is the per-capita birth rate of mated females, which is species-independent. Assume also that mortality is species-independent but sex-specific, with $d_{m}$ and $d_{f}$ as the per-capita, density-independent mortality rates of males and females, respectively. To account for intra- and inter-specific density-dependent feedbacks due to competition, let $a_{i j}$ be the strength of the competitive effect of species $j$ on species $i$. For simplicity, we assume these density-dependent effects are not sex-specific. However, the model can be easily modified to account for these sex-specific feedbacks. Under these assumptions, the model is

$$
\begin{aligned}
\frac{d f_{i}}{d t} & =f_{i}\left(b \frac{m_{i}}{f_{i}+m_{i}}-d_{f}-\sum_{j} a_{i j}\left(m_{j}+f_{j}\right)\right) \\
\frac{d m_{i}}{d t} & =m_{i}\left(b \frac{f_{i}}{f_{i}+m_{i}}-d_{m}-\sum_{j} a_{i j}\left(m_{j}+f_{j}\right)\right) \\
i & =1,2,3
\end{aligned}
$$


To ensure each species can persist in the absence of the others, we assume that $b>d_{m}+d_{f}$. To account for rock-paper-scissors competitive dynamics, we assume the interaction terms $a_{i j}$ are given by

$$
A=a+\left(\begin{array}{ccc}
0 & \beta & -\alpha \\
-\alpha & 0 & \beta \\
\beta & -\alpha & 0
\end{array}\right)
$$

where $a, \alpha, \beta$ are all positive and $\alpha<a$.

Due to the frequency dependent terms, this model does not satisfy the continuity assumptions of Hofbauer and Schreiber (2010) and, consequently, their results can not be applied directly to study permanence of these equations. However, through the change of variables,

$$
x_{i}=m_{i}+f_{i} \text { and } y_{i}=\frac{f_{i}}{x_{i}}
$$

Equation (16) transforms to

$$
\begin{aligned}
\frac{d x_{i}}{d t} & =x_{i}\left(2 b y_{i}\left(1-y_{i}\right)-d_{f} y_{i}-d_{m}\left(1-y_{i}\right)-\sum_{j} a_{i j} x_{j}\right) \\
\frac{d y_{i}}{d t} & =y_{i}\left(1-y_{i}\right)\left(b+d_{m}-d_{f}-2 b y_{i}\right) \\
i & =1,2,3
\end{aligned}
$$

and our permanence theorem applies to prove

Theorem 5 Under these assumptions, if $\alpha>\beta$, then (17) is permanent in $\mathbb{R}_{+}^{3} \times$ $(0,1)^{3}$. Conversely, if $\alpha<\beta$, then (17) is not permanent.

Proof First, note that (17) satisfies the assumptions of our main theorem (1). The dynamics on the extinction set consist of an unstable equilibrium at $(x, y)=(0,0,0) \times$ $\left(\hat{y}_{1}, \hat{y}_{2}, \hat{y}_{3}\right)$ and a heteroclinic cycle between single species equilibria (e.g. $\left(\hat{x}_{1}, 0,0\right) \times$ $\left.\left(\hat{y}_{1}, \hat{y}_{2}, \hat{y}_{3}\right)\right)$ where

$$
\hat{x}_{i}=\frac{b-d_{m}-d_{f}}{a}, \hat{y}_{i}=\frac{1}{2}+\frac{d_{m}-d_{f}}{2 b} \text { and } x_{j}=y_{j}=0 \text { for } j \neq i .
$$

At these equilibria, the per-capita growth rates of the missing species are $\alpha \hat{x}_{i}$ and $-\beta \hat{x}_{i}$. Using the Morse decomposition consisting of the zero equilibrium and the heteroclinic cycle, Theorem 1 implies that permanence occurs if there exist $p_{i}>0$ such that

$$
\begin{aligned}
& p_{1} \cdot 0+p_{2} \cdot \alpha \hat{x}_{1}+p_{2} \cdot\left(-\beta \hat{x}_{1}\right)>0 \\
& p_{1} \cdot\left(-\beta \hat{x}_{2}\right)+p_{2} \cdot 0+p_{2} \cdot \alpha \hat{x}_{2}>0 \\
& p_{1} \cdot \alpha \hat{x}_{3}+p_{2} \cdot\left(-\beta \hat{x}_{3}\right)+p_{2} \cdot 0>0 .
\end{aligned}
$$


As $\hat{x}_{1}=\hat{x}_{2}=\hat{x}_{3}$, there is a solution to these linear inequalities if and only if $\alpha>\beta$. Conversely, there is a solution to the reversed linear inequalities if and only if $\beta>\alpha$ and then Theorem 1 implies that (17) is not permanent.

Theorem 5 yields the same permanence condition as in the classic asexual model. Due to our assumption that density-dependent feedbacks are not sex-specific, the system is only partially coupled as the frequency dynamics of $y$ do not depend on $x$. With sex-specific density-dependent feedbacks, the system would be fully coupled but still analytically tractable as these feedbacks would appear as linear functions of $x_{i}$ in the $y_{i}$ equations.

\subsection{Quantitative genetics}

In recent years, empirical studies have demonstrated that feedbacks between evolutionary and ecological processes (eco-evolutionary feedbacks) can affect coexistence of species (Lankau and Strauss 2007). As a consequence of the growing empirical evidence, theoreticians have developed models that couple commonly used ecological models with evolutionary equations to study eco-evolutionary feedbacks. For the evolution of quantitative traits, such as body size, a common approach is to assume that the rate of trait change is proportional to the gradient of per-capita growth with respect to the trait (Lande 1976). This has led to models of the form

$$
\begin{aligned}
\frac{d x_{i}}{d t} & =x_{i} f_{i}(x, y) \quad i=1 \ldots n \\
\frac{d y}{d t} & =\sigma_{G}^{2} \frac{\partial f_{j}}{\partial y}
\end{aligned}
$$

where $y$ represents the mean of an evolving quantitative trait of one of the species $j$, and $\sigma_{G}^{2}$ is the heritable variance of the trait (Lande 1976). These feedbacks are immediately in the form of (2) and we can use Theorem 1 to identify when ecoevolutionary feedbacks mediate coexistence.

For illustrative purposes, we consider a model developed by Schreiber et al. (2011b). They consider the apparent competition community module, in which two prey species with densities $x_{1}, x_{2}$, respectively, share a common predator with density $x_{3}$. In this model, the predator population has a quantitative trait that determines the attack rate of individual predators on each prey species. The quantitative trait is assumed to be normally distributed with variance $\sigma$ in the predator population with mean $y \in\left[\theta_{1}, \theta_{2}\right]$, where $\theta_{i}$ is the optimal trait for attacking prey $i$. They derived a function $a_{i}(y)$ of the average attack rate of the predator on prey $i$ that decreases with the distance between the trait $y$ and $\theta_{i}$, given by

$$
a_{i}(y)=\frac{\alpha_{i} \tau}{\sqrt{\sigma^{2}+\tau^{2}}} \exp \left[-\frac{\left(y-\theta_{i}\right)^{2}}{2\left(\sigma^{2}+\tau^{2}\right)}\right]
$$


where $\alpha_{i}$ is the maximum attack rate on prey $i$ and $\tau>0$ determines how specialized the predator must be to attack each prey. The coupled dynamics are

$$
\begin{aligned}
\frac{d x_{i}}{d t} & =x_{i}\left(r_{i}\left(1-x_{i} / K_{i}\right)-x_{3} a_{i}(y)\right) \quad i=1,2 \\
\frac{d x_{3}}{d t} & =x_{3} f_{3}(x, y) \\
\frac{d y}{d t} & =\sigma_{G}^{2} \frac{\partial f_{3}}{\partial y}
\end{aligned}
$$

where $K_{i}>0$ and $r_{i}>0$ are the carrying capacity and intrinsic growth, respectively, for prey $i . f_{3}$ is the average per-capita growth rate or fitness of the evolving species given by,

$$
f_{3}(x, y)=\sum_{i=1}^{2} e_{i} a_{i}(y) x_{i}-d
$$

where $e_{i}>0$ is the efficiency at which the predator converts prey $i$ into new predators and $d>0$ is the intrinsic death rate of the predator.

We can apply Theorem 1 to characterize permanence of this system.

Theorem 6 Let $W=\left\{y \in\left[\theta_{1}, \theta_{2}\right] \mid \frac{\partial f_{3}}{\partial y}\left(K_{1}, K_{2}, y\right)=0\right\}$ be the set of equilibria for the trait dynamics when the prey are at carrying capacity and the predator density is zero. If

1. $\frac{r_{i}}{a_{i}\left(\theta_{j}\right)}>\frac{r_{j}}{a_{j}\left(\theta_{j}\right)}\left(1-\frac{d}{a_{j}\left(\theta_{j}\right) e_{j} K_{j}}\right)$ for $i=1,2 ; i \neq j$ and

2. $e_{1} a_{1}\left(y^{*}\right) K_{1}+e_{2} a_{2}\left(y^{*}\right) K_{2}>d$ for all $y^{*} \in W$

then the system is robustly permanent in $\mathbb{R}_{+}^{3} \times\left[\theta_{1}, \theta_{2}\right]$. Conversely, if any inequality is reversed, then the system is not permanent.

The first condition ensures that prey species $i$ has positive per-capita growth when the predator has evolved to optimize on prey $j \neq i\left(y=\theta_{j}\right)$ and the predator and prey $j$ are at their unique equilibrium densities. The second condition ensures that when the predator is rare and both prey are at carrying capacity, the predator has positive growth when it evolves to one of its trait equilibria. Using a different approach, Schreiber and Patel (2015) show (19) is permanent under these conditions. Our results strengthen their results by showing robust permanence.

Proof Equation (19) satisfies the assumptions of Theorem 1. In particular, there is a global attractor $\Gamma$. Let $M_{6}=\{(0,0,0)\} \times\left[\theta_{1}, \theta_{2}\right], M_{5}=\left\{\left(K_{1}, 0,0, \theta_{1}\right)\right\}, M_{4}=$ $\left\{\left(0, K_{2}, 0, \theta_{2}\right)\right\}, M_{3}=\left\{\left(\hat{x}_{1}, 0, \hat{x}_{3}^{(1)}, \theta_{1}\right)\right\}$, and $M_{2}=\left\{\left(0, \hat{x}_{2}, \hat{x}_{3}^{(2)}, \theta_{2}\right)\right\}$ where $\hat{x}_{i}=$ $\frac{d}{e_{i} a_{i}\left(\theta_{i}\right)}$ and $\hat{x}_{3}^{(i)}=\frac{r_{i}\left(1-\frac{\hat{x}_{i}}{K_{i}}\right)}{a_{i}\left(\theta_{i}\right)}$. Finally, let $M_{1}=\left\{\left(K_{1}, K_{2}, 0\right)\right\} \times\left[y_{1}, y_{2}\right]$ where $y_{1}=$ $\min _{y \in W} y$ and $y_{2}=\max _{y \in W} y$. Schreiber and Patel (2015) consider three separate cases: (i) $d \geq a_{1}\left(\theta_{1}\right) e_{1} K_{1}$, (ii) $a_{1}\left(\theta_{1}\right) e_{1} K_{1}>d \geq a_{2}\left(\theta_{2}\right) e_{2} K_{2}$ or (iii) $a_{2}\left(\theta_{2}\right) e_{2} K_{2}>$ $d$. They show that $\mathcal{M}_{1}=\left\{M_{1}, M_{4}, M_{5}, M_{6}\right\}, \mathcal{M}_{2}=\left\{M_{1}, M_{3}, M_{4}, M_{5}, M_{6}\right\}$ and 
$\mathcal{M}_{3}=\left\{M_{1}, M_{2}, M_{3}, M_{4}, M_{5}, M_{6}\right\}$ form a Morse decomposition for (19) under case (i), (ii), and (iii) restricted to $\Gamma \cap S_{0}$, respectively.

Consider case (iii). For each Morse set $M_{k} \in \mathcal{M}_{3}$, there exist a vector $p_{k}$ that satisfies the inequality in Theorem 1 for every point in the set. For example, for $\epsilon$ sufficiently small, $p_{6}=(1,1, \epsilon)$ satisfies the inequality in Theorem 1 for $M_{6}$. Case (ii) and case (i) follow similarly.

\section{Discussion}

Understanding how abiotic and biotic factors determine coexistence of interacting species is a fundamental problem in ecology. Ecologists have demonstrated that factors internal to the populations, such as individual variation (Bolnick et al. 2011; Violle et al. 2012; Hart et al. 2016; Barabás and D’Andrea 2016) and evolution (Lankau and Strauss 2007; Barabás and D’Andrea 2016), and factors external to the populations, such as temporal variation in abiotic factors (Hutchinson 1961), can have substantial impacts on population dynamics. Moreover, as these internal and external factors change, their influence on population growth leads to changes in population densities which in turn may alter these factors, thereby creating a feedback loop. The instrumental role of this feedback on coexistence has been demonstrated both empirically (e.g. Lankau and Strauss 2007; Chung and Rudgers 2016) as well as theoretically (e.g. Bever et al. 1997; Revilla et al. 2013). Our work develops the mathematical framework for finding conditions that enable coexistence in community dynamic models with feedbacks and, by applying this theory, elucidates the role of these internal and external feedbacks on coexistence.

We find that if there is a weighting of the species such that the temporal average of the weighted per-capita growth rates is positive whenever a species is missing, then populations coexist with feedbacks. Moreover, given a Morse decomposition for the extinction set, these weightings can differ among the components of this decomposition. For models without feedbacks, this sufficient condition for robust permanence is equivalent to the condition found by Garay and Hofbauer (2003). Hence, our results provide a natural extension to models with internal and external feedbacks. As our examples illustrate, these feedbacks play two critical roles for coexistence. First, the effect of the feedback variable will influence the Morse decomposition for the extinction set. Second, feedbacks affect the per-capita growth of each of the species and thereby, influence whether the weighted combination of these growth rates can be positive. These differences can drive feedbacks to enable or prevent coexistence.

In addition to extending the work of Garay and Hofbauer (2003) to include internal and external feedbacks, our general framework and permanence result incorporates existing population models with specific types of internal feedbacks (Caswell and Weeks 1986; Caswell 2001; Hofbauer and Schreiber 2010), external feedbacks (Armstrong and McGehee 1976; Mottoni and Schiaffino 1981; Zhao 2001), and mixtures of internal and external feedbacks (Hastings et al. 2007; Cuddington et al. 2009). Through our examples, we illustrate how to transform several of these earlier results into our framework. In our first example, we formulate a non-autonomous model with parameters that vary with the environment into our framework by introducing 
a feedback variable that models the dynamics of the environmental variation. In the second example, we transform a structured population model, in which populations are partitioned into distinct types, into our framework, via a change of variables into frequencies of types and total densities. The frequencies of the different types within the population act as the internal feedback variables. Finally, in the third example, we demonstrate how our results apply to population models with feedbacks due to trait evolution. These examples highlight that our framework can help elucidate how populations coexist in a range of ecological scenarios.

In an attempt to explain empirical evidence for coexistence that was incompatible with theoretical predictions, Hutchinson (1961) postulates that changes in the environment that alter the competitive superiority of one species over another can enable coexistence. Our first example highlights that ergodic environmental variation that drives temporal differences in ecological parameters can enable coexistence of populations in a community, but that this depends on the role it has on influencing population growth. In particular, we show that environmental variation that influences species interactions enables coexistence, in comparison to an analogous model that uses time-averaged parameters instead of explicitly accounting for variation. Our results are an extension of previous work that showed coexistence amongst two competing species with periodic environmental variation (Armstrong and McGehee 1976; Cushing 1980; Mottoni and Schiaffino 1981) and a specification of the general results for non-autonomous two species models (Zhao 2001). Notably, our example demonstrates how variation and separation of resource use between two species can enable coexistence through a storage effect (Chesson and Warner 1981), provided that species are "stored" through periods they do not use the resource and can sufficiently recover through periods in which they do. Interestingly, our results also highlight that environmental variation that only influences non-interaction terms, such as per-capita mortality, does not enable coexistence due to the linearity of noninteraction terms in the model. The necessity for temporal variation to act in nonlinear ways to enable coexistence was also noted for models with stochastic environments (Schreiber 2010) as well as in discrete time models with non-overlapping generations (Chesson and Warner 1981; Chesson 1994).

In addition to externally-driven temporal variation, internal variation within populations may also impact coexistence. Many reviews highlight that models with internal variation can lead to different predictions and inferences in both empirical and theoretical ecological studies compared to mean field models (Bolnick et al. 2011; Violle et al. 2012; Hart et al. 2016). Structured population models, a commonly used framework for accounting for internal variation, involve partitioning the population into distinct types, such as based on sex, life stages, or location in space, so that each type has its own growth rate depending on all other types (Caswell and Weeks 1986; Caswell 2001). Our permanence condition can be used to determine when structured interacting populations coexist. These results apply to structured models that previous results from Hofbauer and Schreiber (2010) do not. Mainly, Hofbauer and Schreiber (2010) made two mathematical assumptions. First, they assume that there were no negative interactions between individuals of different types. This assumption may not hold in a number of common ecological scenarios, including models with cannibalism or other forms of interference, which is a prevalent negative interaction between different 
life stages within a population (Fox 1975; Polis 1981). Second, they assume continuity in the growth matrices $A_{i}$, which restricts their framework to models with no frequency dependent growth. Growth in structured models may be frequency dependent in a number of biological scenarios. In our example, we apply our results to a sexually-structured model, which, following Caswell and Weeks (1986), has frequency dependence since fecundity depends on sex ratios. In particular, through a change of variable from the densities of different types within a species to total density and frequency of types, structured models can be reformulated into our framework, making the permanence conditions applicable to a broad range of structured models.

When individual variation in a population is heritable, this sets the stage for evolution to take place in response to differential selection pressures (Violle et al. 2012). Recent empirical evidence has demonstrated the prevalence of feedbacks between population dynamics and trait evolution (called eco-evolutionary feedbacks; reviewed in Schoener 2011; Lankau and Strauss 2011 and that these feedbacks may impact population dynamics (Abrams 2000; Lankau 2009; Cortez and Ellner 2010; Vasseur et al. 2011; Schreiber et al. 2011b; Northfield and Ives 2013; Patel and Schreiber 2015). Thus far, few studies have shown permanence in these types of models (but see (Schreiber et al. 2011b; Schreiber and Patel 2015)), and we hope that these results will motivate analyses of coexistence in the sense of permanence in future eco-evolutionary studies. Through our example, we demonstrate how these results can elucidate the conditions for robust permanence in a model where a predator is evolving between traits that are more fit for attacking one prey species versus another. In the absence of ecoevolutionary feedbacks, the prey species exhibit apparent competition: increasing the density of one prey increases the predator density and, thereby, results in a reduction of the other prey species (Holt 1977). For highly enriched environments in which the carrying capacities of the prey are large, this apparent competition can lead to exclusion of one of the prey species (Holt and Lawton 1994). As the predator evolves to specialize on the more common prey, eco-evolutionary feedbacks can rescue the rare prey from this outcome and enable coexistence. Applying our results to other ecoevolutionary models may provide opportunities to gain a more general understanding of the role of evolution on species coexistence.

Our results here extend existing methods for permanence to account for internal and external feedbacks, generalizing some existing results and broadening their applicability. There are a number of natural avenues that would be useful to develop in the future, including infinite dimensional models and stochastic models. We assume feedbacks are contained in $\mathbb{R}_{+}^{m}$. However, some internal and external feedbacks may be better captured in infinite dimensions and extending our results to account for this may be useful (e.g. integral projection models; Easterling et al. 2000). Permanence has been studied in general infinite dimensional dynamical systems (Hale and Waltman 1989; Smith and Thieme 2011) as well as in models with specific types of feedbacks, including those captured through continuous spatial heterogeneity (Dunbar et al. 1986; Cantrell et al. 1993, 1996; Zhao and Hutson 1994; Furter and López-Gómez 1997; Cantrell and Cosner 2003; Zhao 2003; Mierczyński et al. 2004; Smith and Thieme 2011), age structure (Smith and Thieme 2011) and time delays (Burton and Hutson 1989; Freedman and Ruan 1995; Ruan and Zhao 1999; Zhao 2003). Whether transforming these models into a framework analogous to the one here is useful requires further 
exploration. Furthermore, populations may feedback with random internal or external factors. Extending permanence results for stochastic population models to account for feedbacks will enable comparisons to our framework to understand broadly the role of random feedbacks on coexistence. With the growing number of empirical studies investigating internal and external factors that influence population dynamics, ecological models are becoming more sophisticated. In order for permanence to remain an important concept in ecology, the methods for demonstrating permanence must continue to expand to these new modeling frameworks.

Acknowledgements Open access funding provided by University of Vienna. Financial support by the U.S. National Science Foundation Grant DMS-1313418 to SJS, the American Association of University Women Dissertation Fellowship to SP and the Austrian Science Fund (FWF) Grant P25188-N25 to Reinhard Burger at the University of Vienna is gratefully acknowledged.

Open Access This article is distributed under the terms of the Creative Commons Attribution 4.0 International License (http://creativecommons.org/licenses/by/4.0/), which permits unrestricted use, distribution, and reproduction in any medium, provided you give appropriate credit to the original author(s) and the source, provide a link to the Creative Commons license, and indicate if changes were made.

\section{Appendix 1: Proof of Theorem 1}

To prove Theorem 1, we begin by introducing "good average Lyapunov functions" and proving a more general theorem.

Definition A1 A continuous map $P: U \rightarrow \mathbb{R}$, where $U \subset S$ is an open set, is a good average Lyapunov function (GALF) for (2) if

- $P(z)=0$ for all $z \in S_{0} \cap U$ and $P(z)>0$ for all $z \in\left(S \backslash S_{0}\right) \cap U$,

- $P$ is differentiable on $\left(S \backslash S_{0}\right) \cap U$,

$-\frac{\partial P}{\partial y_{j}}=0$ for all $j$,

$-p_{i}:=\frac{x_{i}}{P} \frac{\partial P}{\partial x_{i}}$, which are continuous functions defined on $\left(S \backslash S_{0}\right) \cap U$ and extend continuously to $S \cap U$, and

- for every $z \in S_{0} \cap U$, there is a $T_{z}>0$ such that $z . t \in U$ for $t \in\left[0, T_{z}\right]$, and

$$
\int_{0}^{T_{z}} \sum_{i} p_{i}(z . t) f_{i}(z . t) d t>0 .
$$

We prove the following theorem

Theorem A1 Let $\mathcal{M}=\left\{M_{1}, M_{2}, \ldots M_{\ell}\right\}$ be a Morse decomposition for $S_{0} \cap \Gamma$ of (2). For each $k$, let $U_{k}$ be an open neighborhood of $M_{k}$ and let $P_{k}: U_{k} \rightarrow \mathbb{R}$ be a good average Lyapunov function for (2). Then (2) is permanent.

Proof Fix $k$. Let $F(z, T):=\int_{0}^{T} \sum_{i} p_{i}(z . t) f_{i}(z . t) d t$ for all $z \in U_{k}$ and $T \geq 0$, where $p_{i}$ is defined from the definition of a GALF.

By definition of the GALF, for all $z \in M_{k}$, there is a $T_{z}>0$ and $\delta(z)>0$ such that

$$
F\left(z, T_{z}\right)>\delta(z)
$$


By continuity of $F$, there is a neighborhood $V_{z} \subset U_{k}$ of $z \in M_{k}$ such that $F\left(v, T_{z}\right)>\frac{\delta(z)}{2}$ for all $v \in V_{z}$. The collection of sets $\left\{V_{z}\right\}_{z \in M_{k}}$ forms an open cover of $M_{k}$. By compactness, there is a finite subcover $\left\{V_{z_{j}}\right\}_{j=1}^{\ell}$. Let $V_{k}=\cup_{j=1}^{\ell} V_{z_{j}}, c=$ $\frac{1}{2} \min \left\{\delta\left(z_{j}\right)\right\}_{j=1}^{\ell}$ and $T=\max \left\{T_{j}\right\}_{j=1}^{\ell}$, where $T_{j}:=T_{z_{j}}$.

Then, $V_{k} \subset U_{k}$ is a neighborhood of $M_{k}$ such that for all $z \in V_{k}$ there is a $0<T_{j}<T$ satisfying

$$
F\left(z, T_{j}\right)>c .
$$

Furthermore, for $z \in\left(S \backslash S_{0}\right) \cap V_{k}$,

$$
\begin{aligned}
\ln \left(P\left(z . T_{j}\right)\right)-\ln (P(z)) & =\int_{0}^{T_{j}} \frac{1}{P(z . s)} \frac{d}{d s} P(z . s) d s \\
& =\int_{0}^{T_{j}} \frac{1}{P(z . s)}\left(\sum_{i=1}^{n} \frac{\partial P}{\partial x_{i}} \frac{d x_{i}}{d s}+\sum_{i=1}^{m} \frac{\partial P}{\partial y_{i}} \frac{d y_{i}}{d s}\right) d s \\
& =\int_{0}^{T_{j}} \sum_{i=1}^{n} p_{i}(z . s) f_{i}(z . s) d s>c,
\end{aligned}
$$

which gives

$$
P\left(z\left(T_{j}\right)\right)>(1+c) P(z) .
$$

By the Corollary to Theorem 2 from Garay (1989), permanence follows from showing that each $M_{k}$ is isolated and that $\left(S \backslash S_{0}\right) \cap W^{s}\left(M_{k}\right)=\emptyset$, where $W^{s}\left(M_{k}\right)=\{z \in$ $\left.S \mid \emptyset \neq \omega(z) \subset M_{k}\right\}$. For any initial condition $z$, let $\gamma^{+}(z)=z \cdot[0, \infty)$ be the forward trajectory of $z$. Assume there is a $z \in\left(S \backslash S_{0}\right) \cap V_{k}$ such that $\gamma^{+}(z) \subseteq V_{k}$. Then, there is a $z^{*} \in \overline{\gamma^{+}(z)} \cap V_{k}$ such that $P\left(z^{*}\right)=\max _{v \in \overline{\gamma^{+}(z)} \cap V_{k}} P(v)$. Then, either (i) there exists a $t^{*}>0$ such that $z^{*}=z . t^{*}$ or (ii) there exists $t_{n} \rightarrow \infty$ such that $z_{n}:=z . t_{n}$ converges to $z^{*}$ as $n \rightarrow \infty$. If (i), then equation (A1) implies $P\left(z^{*} \cdot T_{z^{*}}\right)>(1+c) P\left(z^{*}\right)$ for some $T_{z^{*}}>0$, which is a contradiction to the choice of $z^{*}$ since $z^{*} \cdot T_{z^{*}} \in \overline{\gamma^{+}(v)}$. If (ii), then for some sequence $T_{z_{n}}>0, P\left(z_{n} . T_{z_{n}}\right)>(1+c) P\left(z_{n}\right) \rightarrow(1+c) P\left(z^{*}\right)$, which is a contradiction to the choice of $z^{*}$ since $z_{n} . T_{z_{n}} \in \overline{\gamma^{+}(z)}$ for all $n$.

Hence, for all $v \in\left(S \backslash S_{0}\right) \cap V_{k}, \gamma^{+}(v) \backslash V_{k} \neq \emptyset$. It follows that $M_{k}$ is isolated and for all $v \in\left(S \backslash S_{0}\right) \cap V_{k}, \omega(v) \not \subset M_{k}$. The latter gives that $\left(S \backslash S_{0}\right) \cap W^{s}\left(M_{k}\right)=\emptyset$.

Theorem 1 immediately follows when using the standard form (4) as a GALF on each Morse set. It is easy to see that when $p_{k i}>0$ for all $i, k,(4)$ satisfies the first four properties of a GALF. In particular, the fourth and fifth property follow since $\frac{x_{i}}{P} \frac{\partial P}{\partial x_{i}}=p_{k i}$ and hence is constant in $U$.

\section{Appendix 2: Proof of Theorem 2}

To show robust permanence, we will use the following theorem from Hirsch et al. (2001) [Corollary 4.5]. Note that this corollary is for maps, but an analogous proof for flows holds (see Hirsch et al. 2001 [Remark 4.6]). 
Theorem $\mathrm{A} 2$ Let $(S, d)$ and $(\Lambda, \rho)$ be metric spaces. For each $\lambda \in \Lambda$, let $\phi_{\lambda}$ : $S \times \mathbb{R} \rightarrow S$ be a flow that is continuous in $\lambda, z, t$. Let $S_{p} \subset S$ be an open subset such that $S_{p}$ is invariant for all $\lambda$ and let $\partial S=S \backslash S_{p}$. Also, assume that every forward trajectory for $\phi_{\lambda}$ has compact closure in $S$ and that $\bigcup_{\lambda \in \Lambda, z \in S} \omega_{\lambda}(z)$ has compact closure, where $\omega_{\lambda}$ denotes the $\omega$-limit for $\phi_{\lambda}$. Let $\lambda_{0} \in \Lambda$ be fixed and assume that

T1: $\phi_{\lambda_{0}}$ has a global attractor $\Gamma$ and there exists a Morse decomposition $\left\{M_{1}, \ldots, M_{\ell}\right\}$ for $\Gamma \cap \partial S$

T2: there exists $\delta_{0}>0$ such that for any $\lambda \in \Lambda$ with $\rho\left(\lambda, \lambda_{0}\right)<\delta_{0}$ and any $z \in S_{p}$, $\lim \sup _{t \rightarrow \infty} d\left(\phi_{\lambda}(z, t), M_{k}\right) \geq \delta_{0}$, for all $1 \leq k \leq \ell$.

Then, there exists $a \beta>0$ and $\delta>0$ such that $\liminf _{t \rightarrow \infty} d\left(\phi_{\lambda}(z, t), \partial S\right) \geq \beta$ for any $\lambda \in \Lambda$ with $\rho\left(\lambda, \lambda_{0}\right)<\delta$ and any $z \in S_{p}$.

To apply this theorem, endow $\Lambda=\Delta(1, Q)$ with the sup norm (i.e. $\rho\left(h_{1}, h_{2}\right)=$ $\left.\left\|\left(h_{1}, h_{2}\right)\right\|_{\infty}=\sup _{z \in Q}\left\|h_{1}(z), h_{2}(z)\right\|\right)$. Let $S=\mathbb{R}^{n+m}$ with the standard metric and let $S_{p}=S \backslash S_{0}$. By $\mathbf{R 3}$ in the definition of perturbations, $\bigcup_{(\tilde{f}, \tilde{g}) \in \Delta(1, Q), z \in S} \widetilde{\omega}(z) \subset Q$ and so has compact closure. For $(f, g)$, we have a Morse decomposition for $\Gamma \cap S_{0}$.

Hence, we only have to show T2. To do this, we show that there exists a $1>\delta>0$ sufficiently small such that if $P_{k}$ is a GALF on $U_{k}$ for (2), then it is also a GALF on $V_{k}$ for every $(\tilde{f}, \tilde{g}) \in \Delta(\delta, Q)$, where $V_{k}$ is defined in the proof of Theorem 1 . First, note that the first four conditions defining a GALF are properties of $P$ and independent of the flow. Hence, these are still satisfied. We must show the final condition: for a $\delta>0$ sufficiently small, for all $z \in V_{k} \cap S_{0}$, there is a $T_{z}>0$ such that

$$
\int_{0}^{T_{z}} \sum_{i=1}^{n} p_{i}(\tilde{z} . t) \tilde{f}_{i}(\tilde{z} . t) d t>0
$$

for every $(\tilde{f}, \tilde{g}) \in \Delta(\delta, Q)$.

Let $F(z, T):=\int_{0}^{T} \sum_{i} p_{i}(z . t) f_{i}(z . t) d t$ and $\tilde{F}(z, T):=\int_{0}^{T} \sum_{i} p_{i}(\tilde{z} . t) \tilde{f}_{i}(\tilde{z} . t) d t$. Let $T, c$ be such that for all $z \in V_{k}$, there is a $0<T_{z}<T$ for which $F\left(z, T_{z}\right)>c$, as in the proof of Theorem 1 . Then, for sufficiently small $\delta>0$,

$$
\begin{aligned}
\left|\tilde{F}\left(z, T_{z}\right)-F\left(z, T_{z}\right)\right| \leq & \int_{0}^{T_{z}} \sum_{1}^{n}\left|p_{i}(\tilde{z} . t) \tilde{f}_{i}(\tilde{z} . t)-p_{i}(\tilde{z} . t) f_{i}(\tilde{z} . t)\right| \\
& +\left|p_{i}(\tilde{z} . t) f_{i}(\tilde{z} . t)-p_{i}(z . t) f_{i}(z . t)\right| d t \\
& <\frac{c}{2}
\end{aligned}
$$

for every $(\tilde{f}, \tilde{g}) \in \Delta(\delta, Q)$ and all $T_{z} \in[0, T]$. The first inequality follows from the triangle inequality. The second inequality follows from $\mathbf{R} 1$ in the definition of perturbations, which constrains the first difference in the sum, and Gronwall's inequality and Lipschitz continuity of $x_{i} f_{i}$ and $g_{i}$, which constrain the second difference in the sum. 
Finally, for all $z \in S_{0} \cap V_{k}$, there is a $0<T_{z}<T$ such that

$$
\tilde{F}\left(z, T_{z}\right) \geq-\left|\tilde{F}\left(z, T_{z}\right)-F\left(z, T_{z}\right)\right|+F\left(z, T_{z}\right) \geq-\frac{c}{2}+c=\frac{c}{2}>0
$$

Hence, for sufficiently small $\delta>0, P_{k}$ is a GALF on isolating neighborhood $V_{k}$ for every $(\tilde{f}, \tilde{g}) \in \Delta(\delta, Q)$. By the same argument as in the proof of Theorem 1 , this implies that for all $z \in S_{p} \cap V_{k}, \gamma^{+}(z) \nsubseteq V_{k}$, which implies T2. Finally, by Theorem A2, every $(\tilde{f}, \tilde{g}) \in \Delta(\delta, Q)$ is permanent and there is a uniform lower bound $\beta>0$ in the definition of permanence for all $(\tilde{f}, \tilde{g})$.

\section{Appendix 3: Proof of Proposition 1}

Proof Choose the trivial Morse decomposition $M:=\left\{\Gamma \cap S_{0}\right\}$. We want to show that for all $z \in M$, there is a $T_{z}$ such that $F\left(z, T_{z}\right)>0$, where $F(z, T):=$ $\int_{0}^{T} \sum_{i} p_{i} f_{i}(z . t) d t$, with $f_{i}(z . t)=\sum_{i} a_{i j} x_{j}+b_{i}$. The proof follows the proof of Theorem 13.6.1 in Hofbauer and Sigmund (1998). For any $I \subseteq\{1, \ldots n\}$, let $\sigma_{I}:=\left\{(x, y) \mid x_{i}>0 \Longleftrightarrow i \in I\right\}$ and let $\ell\left(\sigma_{I}\right)$ be the cardinality of $I$. The $\sigma_{I}$ are invariant. The proof is by induction. First, assume $z \in \sigma_{I}$ with $\ell\left(\sigma_{I}\right)=0$, so that $z=(0, y)$. Then, by inequality (9), $\sum_{i} p_{i} \bar{b}_{i}>0$. Hence, there is a $T_{z}$ such that $F\left(z, T_{z}\right)>0$.

Now, assume that for all $z \in \sigma_{I}$ such that $\ell\left(\sigma_{I}\right) \leq k-1$, there is a $T_{z}$ such that $F\left(z, T_{z}\right)>0$. Let $z$ be a point in $\sigma_{J}$ with $\ell\left(\sigma_{J}\right)=k$. There are two possible cases. In case $1, \omega(z) \subset \bigcup_{\ell\left(\sigma_{I}\right) \leq k-1} \sigma_{I} \cap M$. As in the proof of Theorem A1, there is a neighborhood $V$ of $\bigcup_{\ell\left(\sigma_{I}\right) \leq k-1} \sigma_{I} \cap M$, a $T>0$ and a $c>0$, such that for all $z \in V$, there is a $T_{z}<T$ such that $F\left(z, T_{z}\right)>c$. Since $\omega(z) \subset \bigcup_{\ell\left(\sigma_{I}\right) \leq k-1} \sigma_{I} \cap M$, there is a $\tilde{T}>0$ such that $z . t \in V$ for all $t>\tilde{T}$. Hence, there is a $T_{z}$ such that $F\left(z, T_{z}\right)>0$

In case $2, \omega(z) \not \subset \bigcup_{\ell\left(\sigma_{I}\right) \leq k-1} \sigma_{I} \cap M$. Then, there exists an $\epsilon$ and an increasing sequence $T_{m} \rightarrow \infty$ such that $x_{i}\left(T_{m}\right)>\epsilon$ for all $i \in J$ and all $T_{m}$. Furthermore, by passing to a subsequence if necessary, $\frac{1}{T_{m}} \int_{0}^{T_{m}} x_{i}(t) d t$ converges to some $\bar{x}_{i}$ for all $i$, as $m \rightarrow \infty$. For $i \notin J, \bar{x}_{i}=0$. By the unique ergodicity of $y, \frac{1}{T_{m}} \int_{0}^{T_{m}} b_{i}(y(t)) d t$ converges to $\bar{b}_{i}$ as $m \rightarrow \infty$.

For $i \in J, \frac{1}{T_{m}}\left[\log x_{i}\left(T_{m}\right)-\log x_{i}(0)\right]=\frac{1}{T_{m}} \int_{0}^{T_{m}} \sum_{j} a_{i j} x_{j}(t)+b_{i}(y(t)) d t$. Since $x_{i}\left(T_{m}\right)>\epsilon$, the left hand side goes to zero as $m \rightarrow \infty$. So for all $i \in J$, $\sum_{j} a_{i j} \frac{1}{T_{m}} \int_{0}^{T_{m}} x_{j}(t) d t+\frac{1}{T_{m}} \int_{0}^{T_{m}} b_{i}(y(t)) d t \rightarrow 0$ as $m \rightarrow \infty$, which implies that $\sum_{j} a_{i j} \bar{x}_{j}=-\bar{b}_{i}$. Finally, by inequality (9), there exists a $T_{z}>0$ such that $\frac{1}{T_{z}} \int_{0}^{T_{z}} \sum p_{i} \sum_{j} a_{i j} x_{j}(t)+b_{i}(y(t)) d t>0$. Applying Theorem 2 concludes that proof.

\section{References}

Abrams PA (2000) The evolution of predator-prey interactions: theory and evidence. Annu Rev Ecol Syst 31:79-105 
Armstrong RA, McGehee R (1976) Coexistence of species competing for shared resources. Theor Popul Biol 9:317-328

Barabás G, D'Andrea R (2016) The effect of intraspecific variation and heritability on community pattern and robustness. Ecol Lett 19:977-986

Bever JD, Westover KM, Antonovics J (1997) Incorporating the soil community into plant population dynamics: the utility of the feedback approach. J Ecol 85:561-573

Bolnick DI, Amarasekare P, Araújo MS, Bürger R, Levine JM, Novak M, Rudolf VH, Schreiber SJ, Urban MC, Vasseur D (2011) Why intraspecific trait variation matters in community ecology. Trends Ecol Evol 26:183-192

Burton T, Hutson V (1989) Repellers in systems with infinite delay. J Math Anal Appl 137:240-263

Butler G, Waltman P (1986) Persistence in dynamical systems. J Differ Equ 63:255-263

Cantrell RS, Cosner C (2003) Spatial ecology via reaction-diffusion equations. Wiley Press, Hoboken

Cantrell RS, Cosner C, Hutson V (1993) Permanence in ecological systems with spatial heterogeneity. Proc R Soc Edinb Sect A Math 123:533-559

Cantrell RS, Cosner C, Hutson V (1996) Ecological models, permanence and spatial heterogeneity. Rocky Mt J Math 26:1-35

Caswell H (2001) Matrix population models. Sinauer Associates, Sunderland

Caswell H, Weeks DE (1986) Two-sex models: chaos, extinction, and other dynamic consequences of sex. Am Nat 128:707-735

Chesson P (1994) Multispecies competition in variable environments. Theor Popul Biol 45:227-276

Chesson PL, Warner RR (1981) Environmental variability promotes coexistence in lottery competitive systems. Am Nat 117:923-943

Chung Y, Rudgers J (2016) Plant-soil feedbacks promote negative frequency dependence in the coexistence of two aridland grasses. Proc R Soc B Biol Sci 283:20160608

Conley C (1978) Isolated invariant sets and the Morse index (CBMS Lecture notes), vol 38. American Mathematical Society, Providence

Cortez MH, Ellner SP (2010) Understanding rapid evolution in predator-prey interactions using the theory of fast-slow dynamical systems. Am Nat 176:E109-E127

Cuddington K, Wilson W, Hastings A (2009) Ecosystem engineers: feedback and population dynamics. Am Nat 173:488-498

Cushing JM (1980) Two species competition in a periodic environment. J Math Biol 10:385-400

Dunbar S, Rybakowski K, Schmitt K (1986) Persistence in models of predator-prey populations with diffusion. J Differ Equ 65:117-138

Easterling M, Ellner S, Dixon P (2000) Size-specific sensitivity: applying a new structured population model. Ecology 81:694-708

Fox LR (1975) Cannibalism in natural populations. Annu Rev Ecol Syst 6:87-106

Freedman H, Ruan S (1995) Uniform persistence in functional differential equations. J Differ Equ 115:173192

Fujiwara M, Pfeiffer G, Boggess M, Day S, Walton J (2011) Coexistence of competing stage-structured populations. Sci Rep 1:107

Furter JE, López-Gómez J (1997) Diffusion-mediated permanence problem for a heterogeneous LotkaVolterra competition model. Proc R Soc Edinb Sect A Math 127:281-336

Garay BM (1989) Uniform persistence and chain recurrence. J Math Anal Appl 139:372-381

Garay BM, Hofbauer J (2003) Robust permanence for ecological differential equations, minimax, and discretizations. SIAM J Math Anal 34:1007-1039

Gatica J, So J (1988) Predator-prey models with almost periodic coefficients. Appl Anal 27:143-152

Gause G (1934) The struggle for existence. Williams and Wilkins, Philadelphia

Hale JK, Waltman P (1989) Persistence in infinite-dimensional systems. SIAM J Math Anal 20:388-395

Hardin G (1960) The competitive exclusion principle. Science 131:1292-1297

Hart SP, Schreiber SJ, Levine JM (2016) Intraspecific variation and species coexistence. Ecol Lett 19:8231006

Hastings A, Byers JE, Crooks JA, Cuddington K, Jones CG, Lambrinos JG, Talley TS, Wilson WG (2007) Ecosystem engineering in space and time. Ecol Lett 10:153-164

Hirsch M, Smith H, Zhao X-Q (2001) Chain transitivity, attractivity, and strong repellors for semidynamical systems. J Dyn Diff Equat 13:107-131

Hofbauer J (1981) A general cooperation theorem for hypercycles. Monatshefte für Mathematik 91:233-240

Hofbauer J, Schreiber SJ (2004) To persist or not to persist? Nonlinearity 17:1393-1406 
Hofbauer J, Schreiber SJ (2010) Robust permanence for interacting structured populations. J Differ Equ 248:1955-1971

Hofbauer J, Sigmund K (1998) Evolutionary games and population dynamics. Cambridge University Press, Cambridge

Holt R (1977) Predation, apparent competition, and the structure of prey communities. Theor Popul Biol 12:197-229

Holt RD, Lawton JH (1994) The ecological consequences of shared natural enemies. Annu Rev Ecol Syst 25:495-520

Hutchinson G (1961) The paradox of the plankton. Am Nat 95:137-145

Hutson V (1984a) Predator mediated coexistence with a switching predator. Math Biosci 68:233-246

Hutson V (1984b) A theorem on average Liapunov functions. Monatshefte für Mathematik 98:267-275

Hutson V (1988) The stability under perturbations of repulsive sets. J Differ Equ 76:77-90

Hutson V, Schmitt K (1992) Permanence and the dynamics of biological systems. Math Biosci 111:1-71

Jansen VA, Sigmund K (1998) Shaken not stirred: on permanence in ecological communities. Theor Popul Biol 54:195-201

Lande R (1976) Natural selection and random genetic drift in phenotypic evolution. Evolution 30:314-334

Lankau RA (2009) Genetic variation promotes long-term coexistence of Brassica nigra and its competitors. Am Nat 174:E40-E53

Lankau RA, Strauss SY (2007) Mutual feedbacks maintain both genetic and species diversity in a plant community. Science 317:1561-1563

Lankau RA, Strauss SY (2011) Newly rare or newly common: evolutionary feedbacks through changes in population density and relative species abundance, and their management implications. Evol Appl 4:338-353

Mierczyński J, Shen W, Zhao X (2004) Uniform persistence for nonautonomous and random parabolic Kolmogorov systems. J Differ Equ 204:471-510

Moll JD, Brown JS (2008) Competition and coexistence with multiple life history stages. Am Nat 171:839843

Mottoni PD, Schiaffino A (1981) Competition systems with periodic coefficients: a geometric approach. J Math Biol 11:319-335

Northfield TD, Ives AR (2013) Coevolution and the effects of climate change on interacting species. PLoS Biol 11:e1001685

Patel S, Schreiber SJ (2015) Evolutionarily driven shifts in communities with intraguild predation. Am Nat 186:E98-E110

Polis GA (1981) The evolution and dynamics of intraspecific predation. Annu Rev Ecol Syst 12:225-251

Revilla TA, Veen G, Eppinga MB, Weissing FJ (2013) Plant-soil feedbacks and the coexistence of competing plants. Theor Ecol 6:99-113

Roth G, Salceanu PL, Schreiber SJ (2017) Robust permanence for ecological maps. SIAM J Math Anal 49:3527-3549

Ruan S, Zhao X (1999) Persistence and extinction in two species reaction-diffusion systems with delays. J Differ Equ 156:71-92

Ruelle D (1979) Analyticity properties of the characteristic exponents of random matrix products. Adv Math 32:68-80

Schoener TW (1976) Alternatives to Lotka-Volterra competition: models of intermediate complexity. Theor Popul Biol 10:309-333

Schoener TW (2011) The newest synthesis: understanding the interplay of evolutionary and ecological dynamics. Science 331:426-429

Schreiber SJ (2000) Criteria for $\mathcal{C}^{r}$ robust permanence. J Differ Equ 162:400-426

Schreiber SJ (2006) Persistence despite perturbations for interacting populations. J Theor Biol 242:844-852

Schreiber SJ (2010) Interactive effects of temporal correlations, spatial heterogeneity and dispersal on population persistence. Proc R Soc B Biol Sci 277:1907-1914

Schreiber SJ, Benaïm M, Atchadé KAS (2011a) Persistence in fluctuating environments. J Math Biol 62:655-683

Schreiber SJ, Bürger R, Bolnick DI (2011b) The community effects of phenotypic and genetic variation within a predator population. Ecology 92:1582-1593

Schreiber SJ, Patel S (2015) Evolutionarily induced alternative states and coexistence in systems with apparent competition. Nat Resour Model 28:475-496 
Smith HL (1995) Monotone dynamical systems. An introduction to the theory of competitive and cooperative systems, volume 41 of mathematical surveys and monographs. American Mathematical Society, Providence

Smith HL, Thieme HR (2011) Dynamical systems and population persistence. American Mathematical Society, Providence

Smith SD, Monson RK, Anderson JE (1997) Physiological ecology of North American desert plants. Springer, New York

Tilman D (1980) Resources: a graphical-mechanistic approach to competition and predation. Am Nat 116:362-393

van Baalen M, Křivan V, van Rijn P, Sabelis M (2001) Alternative food, switching predators, and the persistence of predator-prey systems. Am Nat 157:512-524

van Leeuwen A, Huss M, Grdmark A, de Roos AM (2014) Ontogenetic specialism in predators with multiple niche shifts prevents predator population recovery and establishment. Ecology 95:2409-2422

Vance R, Coddington E (1989) A nonautonomous model of population growth. J Math Biol 27:491-506

Vasseur DA, Amarasekare P, Rudolf VH, Levine JM (2011) Eco-evolutionary dynamics enable coexistence via neighbor-dependent selection. Am Nat 178:E96-E109

Violle C, Enquist BJ, McGill BJ, Jiang L, Albert CH, Hulshof C, Jung V, Messier J (2012) The return of the variance: intraspecific variability in community ecology. Trends Ecol Evol 27:244-252

Volterra V (1928) Variations and fluctuations of the number of individuals in animal species living together. J Cons 3:3-51

Zhao X-Q (2001) Uniform persistence in processes with application to nonautonomous competitive models. J Math Anal Appl 258:87-101

Zhao X-Q (2003) Dynamical systems in population biology. Springer, New York

Zhao X-Q, Hutson V (1994) Permanence in Kolmogorov periodic predator-prey models with diffusion. Nonlinear Anal Theory Methods Appl 23:651-668 